\title{
3D characterization of rolling contact fatigue crack networks
}

Jessop, Casey; AhIström, Johan; Hammar, Lars; Fæster, Søren; Danielsen, Hilmar Kjartansson

Published in:

Wear

Link to article, DOI:

10.1016/j.wear.2016.06.027

Publication date:

2016

Document Version

Peer reviewed version

Link back to DTU Orbit

Citation (APA):

Jessop, C., Ahlström, J., Hammar, L., Fæster, S., \& Danielsen, H. K. (2016). 3D characterization of rolling contact fatigue crack networks. Wear, 366-367, 392-400. https://doi.org/10.1016/j.wear.2016.06.027

\section{General rights}

Copyright and moral rights for the publications made accessible in the public portal are retained by the authors and/or other copyright owners and it is a condition of accessing publications that users recognise and abide by the legal requirements associated with these rights.

- Users may download and print one copy of any publication from the public portal for the purpose of private study or research.

- You may not further distribute the material or use it for any profit-making activity or commercial gain

- You may freely distribute the URL identifying the publication in the public portal

If you believe that this document breaches copyright please contact us providing details, and we will remove access to the work immediately and investigate your claim 


\title{
3D characterization of rolling contact fatigue crack networks
}

\author{
Casey Jessop a , Johan Ahlström ${ }^{\text {a }}$, Lars Hammar ${ }^{\text {a }}$, Søren Fæster ${ }^{\text {b }}$, Hilmar K. Danielsen ${ }^{\text {b }}$ \\ a Department of Materials and Manufacturing Technology, Chalmers University of Technology, Gothenburg, Sweden \\ b Department of Wind Energy, Technical University of Denmark, Risø Campus, Denmark
}

\section{A R T I C L E I N F O}

\section{Article history:}

Received 5 October 2015

Received in revised form

28 June 2016

Accepted 30 June 2016

\section{Keywords:}

Rolling contact fatigue

Optical microscopy

Electron microscopy

Profilometry

Radiography

X-ray tomography

\begin{abstract}
A B S T R A C T
Rolling contact fatigue (RCF) damage is becoming more frequent with increased traffic, accelerations, and loading conditions in the railway industry. Defects which are characterized by a two-lobe darkened surface and a V-shaped surface-breaking crack are defined as squats. The origination and propagation of squats in railway rails is the topic of many recent studies; the associated crack networks develop with complicated geometry near the surface of rails, but can be difficult to detect and distinguish from normally existing head checks in their early stages, using in-field non-destructive detection techniques. After cutting out damaged sections of rail, there are a number of options to characterize the damage. The aim of this study was to evaluate different methods to geometrically describe squat crack networks; through X-ray radiography complemented with geometrical reconstruction, metallography, X-ray tomography, and topography measurements. The experiments were performed on squats from rail sections taken from the field. In the first method, high-resolution and high-energy X-ray images exposed through the entire rail head from a range of angles were combined using a semi-automated image analysis method for geometrical reconstruction, and a 3D representation of the complex crack network was achieved. This was compared with measurements on cross-sections after repeated metallographic sectioning to determine the accuracy of prediction of the geometrical reconstruction. A second squat was investigated by X-ray tomography after extraction of a section of the rail head. A third squat was opened by careful cutting, which gave full access to the crack faces, and the topography was measured by stylus profilometry. The high-energy X-ray, 3D reconstruction method showed accurate main crack geometry at medium depths; the advantage of the method being that it potentially could be developed for nondestructive testing in field. However significant drawbacks exist due to limitations in radiography in terms of detecting tightly closed cracks in very thick components. This includes the inability to detect the crack tips which is an important factor in determining the risks associated to a specific crack. Metallographic investigation of the cracks gave good interpretation of crack geometry along the sections examined, and gave the possibility to study microstructure and plastic deformation adjacent to the crack face. However this time-consuming method requires destruction of the specimen investigated. The X-ray tomography revealed the 3D crack network including side branches in a $10 \times 10 \times 30 \mathrm{~mm}^{3}$ sample, and provided topographic information without completely opening the squat. Topography measurements acquired by stylus profilometry provided an accurate description of the entire main crack surface texture, including features such as surface ridges and beach marks.
\end{abstract}

(c) 2016 Elsevier B.V. All rights reserved.

\section{Introduction}

Heavier loads and increased accelerations and speeds on today's railways are causing increasing damage to rails and wheels [1-3]. The early detection and correct categorization of surface and near-surface defects could help reduce the maintenance costs as well as frequency and intensity of failures and accidents. In order to develop a proper maintenance system, it is important to firstly understand the nature, causes, and consequences of different

E-mail address: casey.jessop@chalmers.se (C. Jessop). defect types. In recent years, squats and squat-like defects have been the subject of several investigations [4-7] and are proving to be an important cause of failure and thus a cost-driver for maintenance worldwide. Squats are a particular type of defect that generally manifest themselves as planar crack networks parallel to the surface within a shallow depth from the rail head; however, in some cases they may develop into cracks perpendicular to the surface, causing transverse rail fracture. Although non-destructive testing techniques could be used to find defects on railway rails on-site, an efficient and reliable on-site non-destructive detection system is not yet available to detect early stages of squat-type 

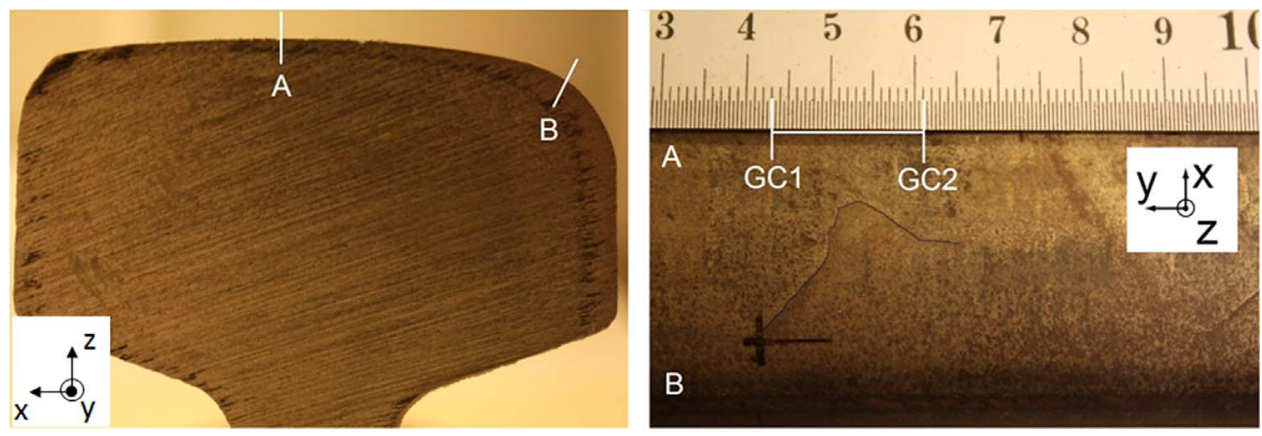

Fig. 1. Squat with gold cross marks (GC1, GC2) and scale (the V-shaped surface-breaking crack has been traced to be more visible).

cracks, since they cannot readily be distinguished from other surface defects which do not have the same associated risks.

\subsection{Squat initiation and growth}

Squats are surface-initiated defects, characterized by their Vshaped surface-breaking crack and two-lobe darkened surface [6], and are classified within three classes: A (light), B (moderate), and C (severe), according to [8,9]. Fig. 1 shows a Category B squat. The initiation of squats is thought to originate due to local plastic deformation of the surface from rolling contact fatigue (RCF) loading, including dynamic wheel-rail contact forces [1]. When local thermal damage on the rail surface is believed to affect the initiation mechanism, the defect is rather referred to as a stud $[1,7]$. In this work we have not determined the cause of initiation due to limited background information, and have chosen to use the name 'squat' for the defects studied.

Although much research is currently being done to identify the early stages of squats, and to prevent their formation (for example, with regular grinding), the development of the crack network of a squat is not well known. The two-lobe form on the surface of a rail suggests a planar crack network below the surface, causing internal wear within the crack, which in turn causes the rail surface to sink and get a darker appearance due to less surface wear. Metallographic cross-sectioning shows an initial crack growing at small angles, around $10^{\circ}$ to $30^{\circ}$, from the surface of the rail, then deviating to propagate nearly parallel to the surface within a shallow depth [10]. In more serious cases, the growth can deviate into a downwards angle leading to risk of rail break [9]. The crack can also branch upwards towards the surface [11], causing spalling which leads to increased impact loading on further traffic.

\subsection{Non-destructive testing of squats}

In early stages of development, RCF defects in rails and wheels are difficult to detect with non-destructive techniques. Eddy current testing, ultrasonic testing, and visual inspection are the most common in-situ inspection techniques in the railway industry $[12,13]$. By the time the crack network can be detected using ultrasonic testing, it could already be a severe squat (for example, it could have traveled up to $4 \mathrm{~mm}$ into the rail) and the rail must be removed. Eddy current testing has the potential for detecting surface and sub-surface defects, but remains extremely sensitive to lift-off [13].

The use of digital radiography is nowadays emerging into other industrial applications, and is promising to be an efficient detection method [14-19]. Radiography of rail heads in field could allow for not only detection of defects, but also a geometrical description of the shape below the surface. Radiographic exposures from several different angles followed by automated image analysis and 3D geometrical reconstruction could be a potential method for non-destructive assessment of the severity of the crack network in situ, but there remain some aspects of the method to develop [19]. Currently, it is being developed in a laboratory environment; however, the goal is to improve the procedure for use on site. Some drawbacks related to the use of radiography still exist, namely with respect to the efficiency of detecting flaws that are not parallel to the radiation, cracks whose orientation require access to difficult positions (for example, longitudinal vertical cracks in rails), small volumes examined per time, and some health and safety concerns.

\subsection{Current study}

In this study, the use of radiography combined with 3D reconstruction, metallography, X-ray tomography, and topography measurement were evaluated as methods to describe the geometry of RCF crack networks. A first squat taken from the field was examined by radiography and metallography, in order to observe the network of cracks, and to investigate the ability of radiography and image analysis to reconstruct the 3D shape. This was verified by comparison with measurements of crack extension on metallographic cross-sections. Metallographic investigation of the material adjacent to the crack faces, was also performed. X-ray tomography was performed on a part of another squat for highresolution 3D visualization of the crack network. A third squat was opened and the topography of the bulk-side crack face was examined by stereomicroscopy and stylus profilometry. The radiography and geometrical reconstruction methods have previously been presented at the 35th Risø symposium on Materials Science and are documented in the proceedings [19] (Fig. 2).

\section{Experimental}

Investigations were carried out on squats taken from different rail sections taken from the field. A first squat was radiographed and thereafter cut in slices which were subjected to metallographic preparation. Before X-ray exposures, reference position indicators (GC1 and GC2 in Fig. 1) were placed on the surface of the rail in order to locate the squat network in 2 dimensions ( $x$ and $y$ ), while a geometrical analysis was done using the radiographs in order to obtain the third dimension $(z)$ required for the $3 \mathrm{D}$ reconstruction of the crack network. The line through the gold crosses corresponds to $x=0 \mathrm{~mm}$, while the center point between GC1 and GC2 defines $y=0 \mathrm{~mm}$. A second squat, taken from a different rail section, was investigated by X-ray tomography. For this, a $10 \mathrm{~mm}$ deep section, $10 \mathrm{~mm}$ along the rolling direction and $30 \mathrm{~mm}$ in the transverse direction, was extracted from the center of the rail running surface (see Fig. 3). A third squat was broken open and the topography of the bulk-side was measured (Fig. 4). 
a

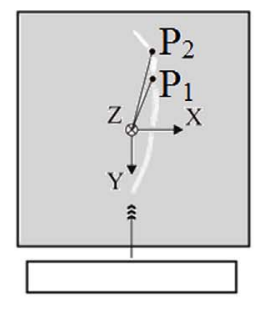

b

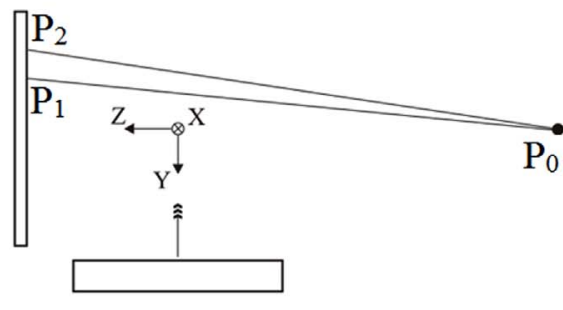

C

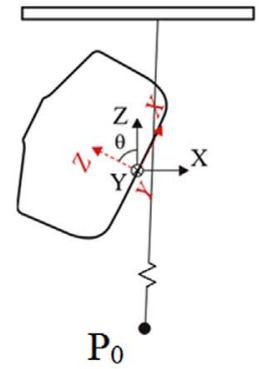

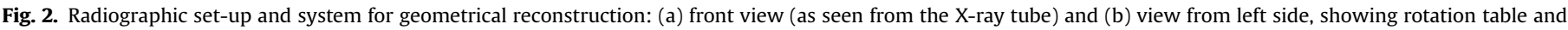

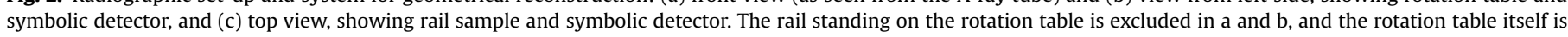
excluded in c for improved visibility.

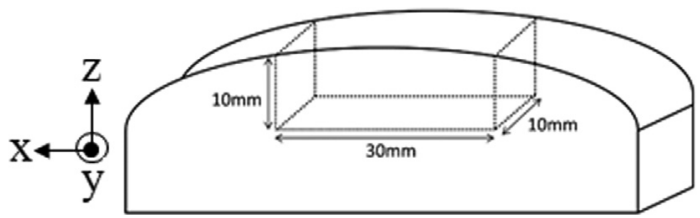

Fig. 3. Dimensions of the sample used for high-resolution 3D X-ray tomography The $\mathrm{y}$-direction is the rolling direction.

\subsection{X-ray imaging technique and image analysis}

X-ray radiography imaging allows for defect detection without destruction of the sample. X-rays penetrate the object, and differences in density along the beam (from cracks and other defects) result in different amounts of absorbed energy, and different shades of gray in the image. The radiography was performed with a GE Titan $450 \mathrm{kV}$ X-ray machine, with constant potential X-ray tube (ISOVOLT $450 \mathrm{M} 2 / 0.4-1.0 \mathrm{HP}$ ). This is a high power tube with a small focus of only $0.4 \mathrm{~mm}$ ( $25 \%$ modulation), and geometric unsharpness of less than $0.06 \mathrm{~mm}$. The X-ray camera is an imaging detector with a scintillating screen and glass fiber optics. A scintillating face plate is used for its unique properties to eliminate the internal light spread and increase the spatial resolution. The detector is Peltier cooled to $-30^{\circ} \mathrm{C}$, and is equipped with a fiber optic input window. The detector is fixed to a front illuminated, full frame 4 Megapixel CCD camera (EEV CCD42-40) with a pixel size of $13.5 \times 13.5 \mu \mathrm{m}^{2}$. The imaging area of the X-ray is $27 \times 27 \mathrm{~mm}^{2}$, with high spatial resolution, measured by unsharpness values less than $0.08 \mathrm{~mm}$ (that is, more than $50 \%$ modulation of the 13th wire pair with the EN ISO 19232-5 double wire IQI). In order to detect the crack within a certain confidence level, image sequences were obtained for exposures in the range of $52-86^{\circ}$, at $2^{\circ}(\Delta \theta)$ intervals. The object is rotated an angle $\Delta \theta$ between each projection, based on the set-up geometry shown in Fig. 2. An accelerating voltage of $400 \mathrm{kV}$, and a $1.75 \mathrm{~mA}$ current were used, with exposure time $8 \times 30 \mathrm{~s}$ ( $7 \mathrm{~mA} \mathrm{~min})$. A deeper description of the radiography method can be found in previous works $[16,17]$.

Each X-ray image is divided into eight exposures, which are integrated to improve the signal-to-noise ratio (SNR). The images may contain radiation-induced noise, therefore each image is statistically compared, and the noise (or white dots) is removed. Conventional background and flat field correction are subsequently done to remove the structural noise. Finally, a "lifting" operation, which compensates for varying thickness of the exposed part of the sample, and thus improves the contrast, is done by multiplying the corrected raw image with an interpolated surface, based on curve fitting in the perpendicular direction of the cracks. The second degree exponential is used as function for curve fitting, as it adapts very well to the line profile of the gray level across the image. In order to better visualize the crack network, the images were also compiled into an animation.

\subsection{Geometrical reconstruction}

The well-established computed tomography (CT) method treated later in this work, allows for automatic evaluation of a 3D crack geometry. However, the tomographic reconstruction ideally requires projections from $360^{\circ}$. Since, in field, the rail is only accessible from the top and sides, conventional tomography is not possible, and a modified geometric reconstruction must be done.

Geometric reconstruction of X-ray images is a method which allows 3D visualization from 2D images, taken in series through an object. For the 3D reconstruction in this study, a combination of visual inspection, a mathematical model, and semi-automated data processing was developed, based on certain characteristics of the radiographs. When parts of the crack are parallel to the Xray beams, good contrast is obtained in the radiograph. The crack plane is thus calculated from the positions where the line profile provides high contrast in the different projections.

The parts of the crack which provide the highest contrast in each image (as projected on the detector) are mapped manually on the images. An illustration is shown in Fig. 2a, where two such points called $P_{1}$ and $P_{2}$ are selected. The path between the points, $\overrightarrow{\mathrm{P}_{1} \mathrm{P}_{2}}$ is assumed to be a straight line and identifies a part of the crack where the radiation is parallel to the crack plane. The larger the curvature as projected on each image, the closer the points are recorded. The plane, $S_{n}$, is obtained from the vector $\overrightarrow{\mathrm{P}_{1} \mathrm{P}_{2_{\mathrm{n}}}}$ and the point $\mathrm{P}_{0}$ positioned in the X-ray source (Fig. $2 \mathrm{~b}$ ). When the object has been rotated by an angle $\Delta \theta$, new points $\mathrm{P}_{1}$ and $\mathrm{P}_{2}$ are selected. A new vector $\overrightarrow{\mathrm{P}_{1} \mathrm{P}_{2_{n}}}$ is obtained, and the next plane, $S_{n+1}$, is calculated. The lines of intersection between the individual planes and the vectors $\overrightarrow{\mathrm{P}_{0} \mathrm{P}_{1}}$ and $\overrightarrow{\mathrm{P}_{0} \mathrm{P}_{2}}$ give the endpoints of each plane. The midpoint between intersection line endpoints is equal to the tangent to the crack plane, which gives the $z$ coordinate of the crack (Fig. 2c). The result was plotted in MATLAB [20].

\subsection{Metallographic cross-sectioning}

In order to evaluate the ability of the X-ray imaging, subsequent image analysis and $3 \mathrm{D}$ reconstruction, to correctly visualize the real 3D geometry of the crack, metallographic crosssectioning was done at different y-positions on the rail sample shown in Fig. 1. The surfaces were prepared by grinding with SiC paper, diamond polishing, and etching with nital $2 \%$. Examination of the prepared surfaces was done using a Zeiss SteREO Discovery V20 stereomicroscope and a Leica light optical microscope (LOM). The series of sections obtained were analyzed using image processing in MATLAB to position the depth of the crack relative to 

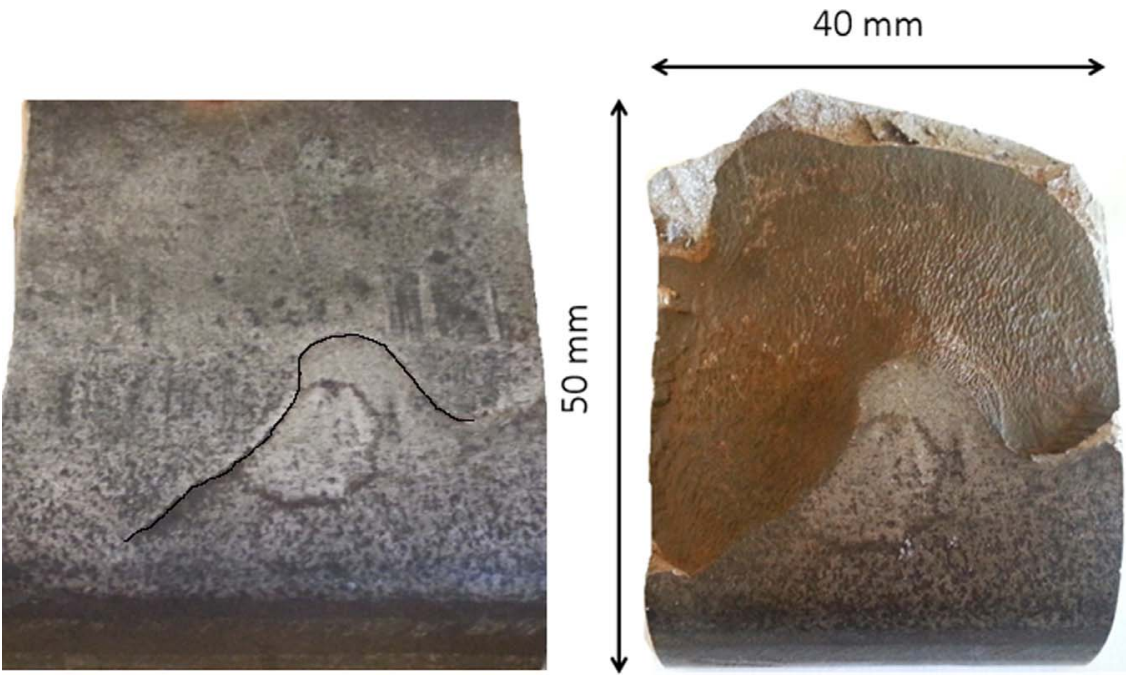

Fig. 4. Category B squat rail surface before (left, slightly tilted) and after (right, showing bulk-side crack face) being opened for topography measurements.

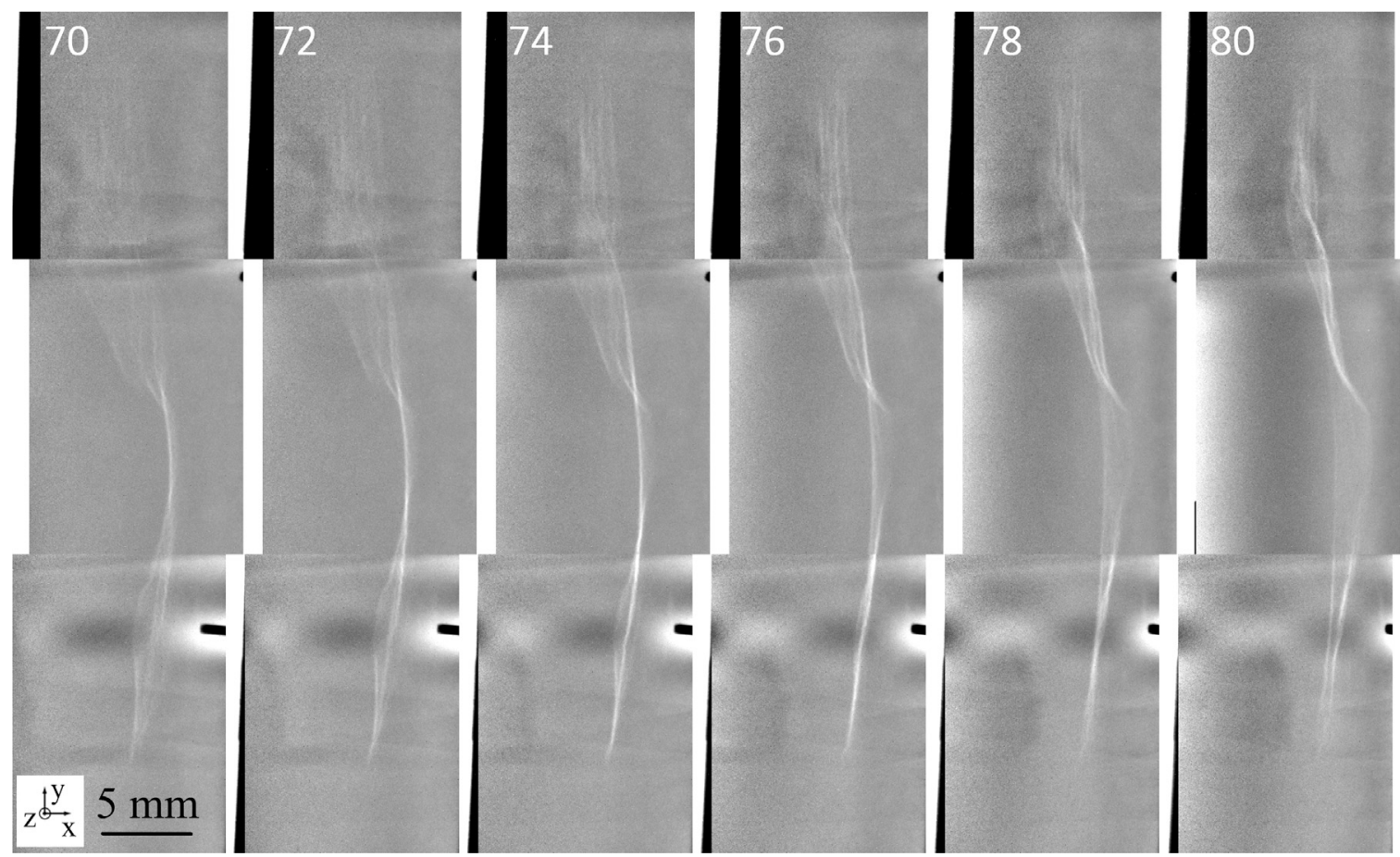

Fig. 5. Superposed X-ray image sequences of squat, ranging from $70^{\circ}$ to $80^{\circ}$.

the surface. The curves resulting from metallography and image analysis were compared to the $3 \mathrm{D}$ reconstruction at given coordinates, to determine the accuracy of the geometric reconstruction method.

Studying the cross-sections with light optical microscopy also gave an overview of the microstructure, including the pearlite lamella orientation and pearlite colony deformation. However, the depth of focus is not sufficient to obtain clear images of a textured surface, requiring the use of scanning electron microscopy (SEM), LEO 1450VP SEM. This technique allows for better spatial resolution, depth of focus, and higher magnification.

\subsection{X-ray tomography}

A sample with dimensions of $10 \times 10 \times 30 \mathrm{~mm}$ containing part of a squat was extracted from the top surface of a rail, see Fig. 3.
Two overlapping regions of the 10 by $10 \mathrm{~mm}^{2}$ cross-section covering the entire sample were scanned tomographically by using a Zeiss Xradia 520. The X-ray energy was polychromatic up to $160 \mathrm{kV}$ from a tungsten target and 1601 projections of each region were acquired. 3D density maps were reconstructed by a standard filtered back-projection method to $2 \mathrm{k} \times 2 \mathrm{k} \times 2 \mathrm{k}$ pixel volumes with a voxel size of $7.288 \mu \mathrm{m}$. Due to some edge effects which have been cut off, the figures only show information from a $8.7 \times 7.3 \times 24.8 \mathrm{~mm}^{3}$ volume.

\subsection{Topography measurements}

A Somicronic Surfascan 3D stylus-based surface profiler was used to obtain 3D mapping of regions of interest on the bulk-side crack surface of a Category B squat. The profiler was fitted with a ST027 stylus probe ( $2 \mu \mathrm{m}$ tip radius and a $90^{\circ}$ tip angle) and a 
depth description was acquired. An overall geometry was obtained using $1 \mathrm{~mm}$ step size over the 40 by $40 \mathrm{~mm}^{2}$ area of the squat (Fig. 4), while detail maps were done on smaller regions with step size $50 \mu \mathrm{m}$ to allow for the ridges to be measured.
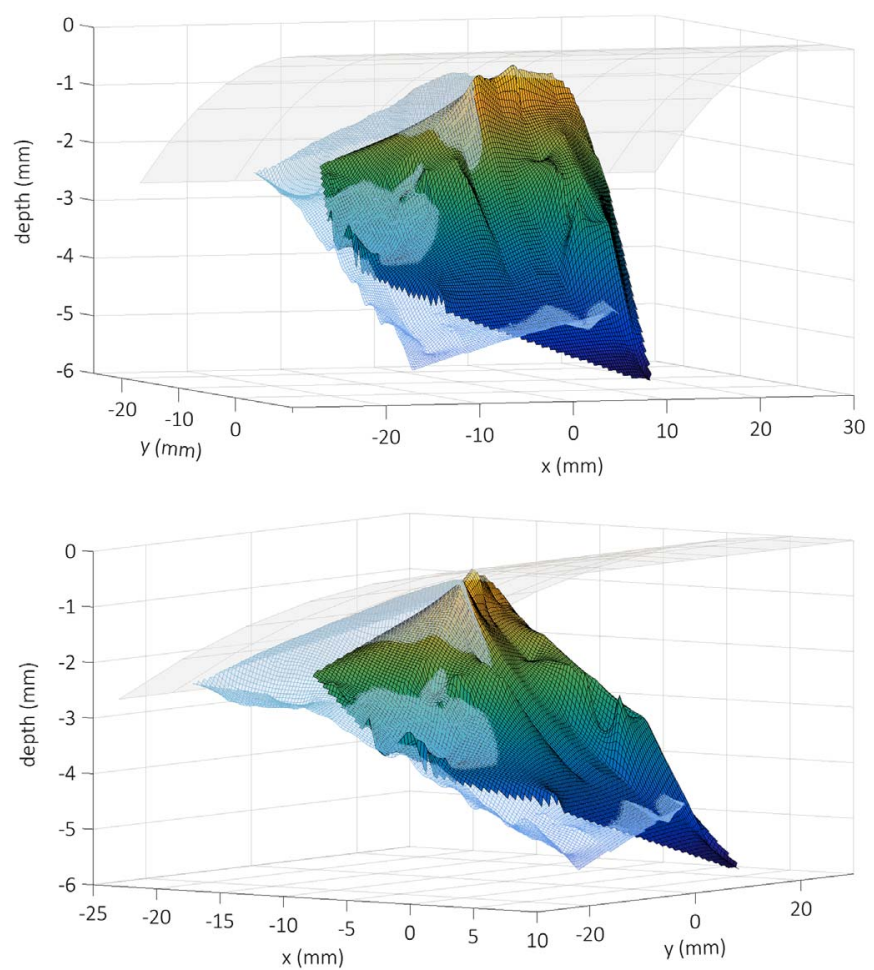

Fig. 6. 3D plots with surfaces obtained from both $3 \mathrm{D}$ reconstruction (colored) and metallography (mesh). The crack network is showed relative to the rail surface (gray). Note that the scaling in z-direction is exaggerated to better show the shape of the crack. (For interpretation of the references to color in this figure legend, the reader is referred to the web version of this article.)

\section{Results}

\subsection{Radiography and image analysis}

The radiographic images obtained after corrections and enhancement allow observation of a given area of the squat crack network, without tampering. Fig. 5 shows a compiled sequence of images from the squat between the angles of +70 and $+80^{\circ}$, with $2^{\circ}$ intervals, according to the previously described radiography set-up (Fig. 2). It can clearly be observed how the intensity increases as the crack normal becomes perpendicular to the incoming X-ray beams. Artefacts are caused by joining images to cover the entire length of the crack, as well as from the necessary mathematical treatment of images. These artefacts currently hinder a fully automated interpretation of images.

\subsection{Geometrical reconstruction}

The result of the 3D geometrical reconstruction is shown in Fig. 6, demonstrating a section of the squat network which is similar to that reported in literature [21].

\subsection{Metallographic cross-sectioning}

Metallographic cross-sectioning was done to determine the accuracy of the geometric reconstruction and also for evaluation of this traditional method as a means to characterise a squat crack. Fig. 7a shows a transverse cross-section of the rail. The comparison between the metallography and the reconstruction shows the reliability of the method for accurate reconstruction of the crack network to a certain extent (Fig. 6), as well as for evaluation of squat severity. It can be seen in the bottom right-hand corner of Fig. 7b that the crack penetrates deeper and further into the rail than seen in the 3D reconstruction, due to the tight closure of the crack tip, making it indistinguishable using radiography through the full rail head. It should be noted that the depth axis is exaggerated in the figures in order to better show the texture of the

\section{a}

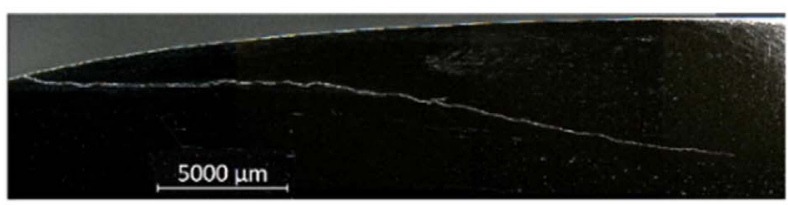

b

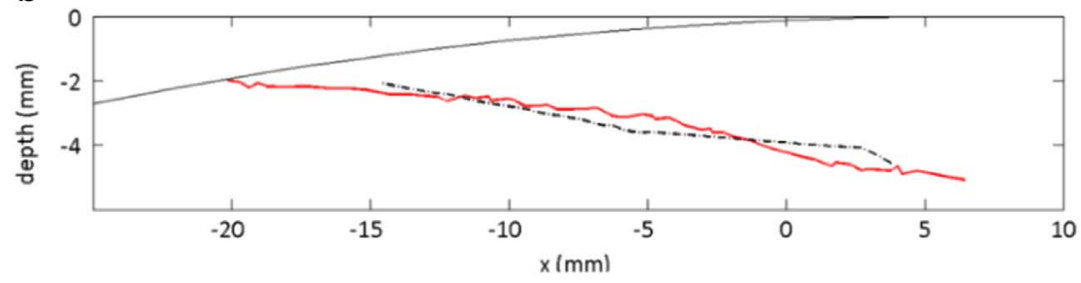

C
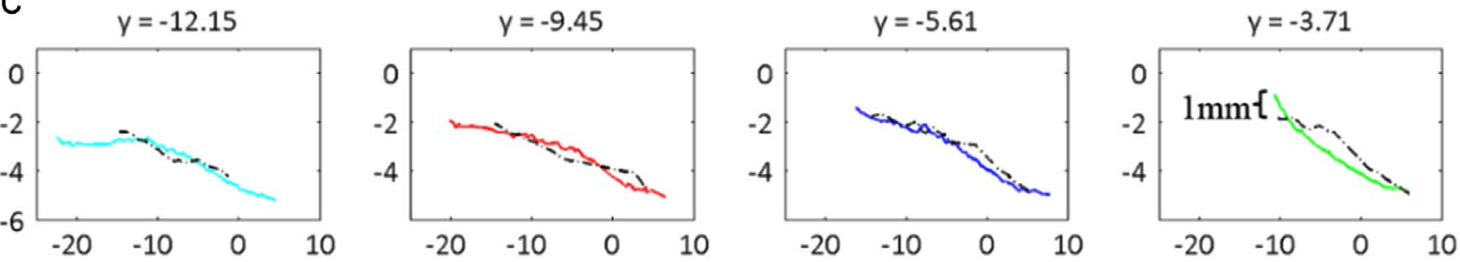

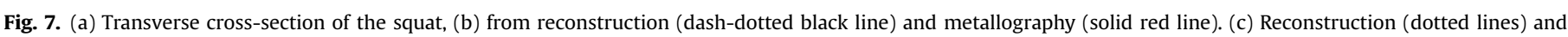

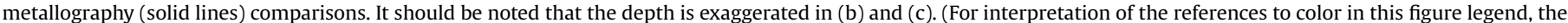
reader is referred to the web version of this article.) 


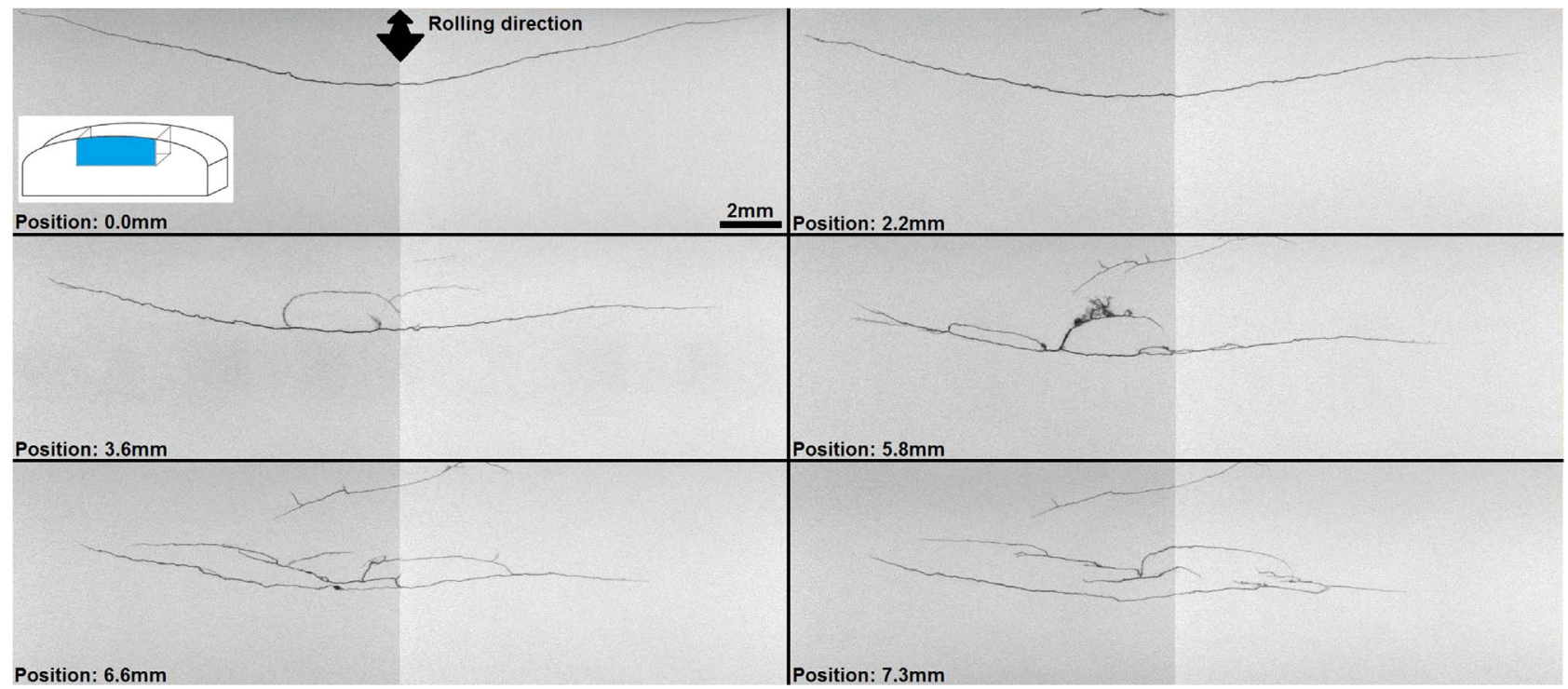

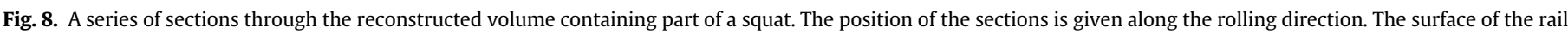
is approximately $400 \mu \mathrm{m}$ above the top of each section.

crack obtained through metallographic sectioning. Similarly, the surface-breaking section of the crack was not always present in the $3 \mathrm{D}$ reconstruction, since planes were plotted assuming piecewise parallelism, there is a limit to the degree of curvature that can be interpreted. The method, however, was found to give a reliable description of the geometry of the crack on medium depths; that is, each curve measured from the metallographic cross-section follows well the interpolated curve from the reconstructed surface, as shown in Fig. 7c.

The effect of plastic deformation on crack propagation was investigated using LOM and SEM. Realignment of pearlite lamellae was apparent at certain areas along the crack, however some areas seemed unaffected. This suggests the crack did not grow following a severely deformed microstructure [22], and that other parameters affected the growth path through the bulk of the material.

\subsection{X-ray tomography}

The 3D density maps obtained by reconstructing X-ray tomography data reveal density differences in the bulk material. The crack network was segmented and visualized by using the Avizo 3D software for materials science. Two overlapping regions were scanned tomographically to cover the squat in the transverse direction. The reconstructed volumes were stitched together and a series of 6 sections through this volume are presented in Fig. 8 along the rolling direction. The two different background contrasts represent the two individual reconstructions. The rolling direction is indicated by an arrow and the surface of the rail is approximately $400 \mu \mathrm{m}$ above the top of the images. Fig. 9 shows one of the scans in the transverse direction. Fig. 10a shows a series of four consecutive sections through a reconstructed volume from the same angle as seen in Fig. 9. Cracks were located automatically by setting an intensity threshold value. Different parts of the 3D structure of the crack network, segmented in blue, are visible in Fig. 9. The same volume is seen from above in Fig. 10b, where part of the fracture surface is magnified. The ridges on the fracture surface of the squat are a characteristic of the RCF crack propagation in the rail. The distance between the ridges has been measured at 10 locations and varies between 200 and $500 \mu \mathrm{m}$.

\subsection{Topography measurements}

An investigation of the topography of the squat on a macro scale was done using stereomicroscopy and stylus profilometry. The fatigue crack path can clearly be followed from the origin to the outside edges, as shown in Fig. 4. Stereomicroscopy images of the same crack are shown in Figs. 11 and 12 shows the mapping from the topography method. A closer examination of the surface (Fig. 11b) shows ridges, very pronounced in certain areas as described by the 3D surface profile in Fig. 13, and occasional beach marks. The mappings obtained, clearly show the texture and roughness of the squat surface, also visible by the X-ray tomography, which are not captured by radiography through the entire rail head nor can be clearly imaged using metallographic sectioning since only one perpendicular plane is imaged.

\section{Discussion}

As the development of RCF defects, and squats in particular, are gaining importance for railway failure prevention, the understanding of their origin, propagation, but also their characterization beyond the surface is necessary. Although squats reveal themselves on the rail surface, it is how they are manifested in a 3D geometry below the surface which characterizes their severity. The replacement of rails is a costly expenditure, and an in-field non-destructive technique would therefore greatly benefit the investigation of squat severity.

\subsection{Radiography and $3 D$ reconstruction}

For the geometrical reconstruction technique, some characteristics of radiography, such as sensitivity to alignment between the $\mathrm{X}$-ray beam and crack, are used in order to obtain a visualization of the complete squat network. It is this characteristic which helps to sort out the normal of the crack plane in each image. Combining information about shape, crack plane orientation, relative movement with respect to the reference position indicators, as well as comparisons between different angles of exposure; a 3D reconstruction is possible. It was found that this radiographic technique provides information on the crack network geometry, however lacks some accuracy in positioning the crack tip, which can be seen 


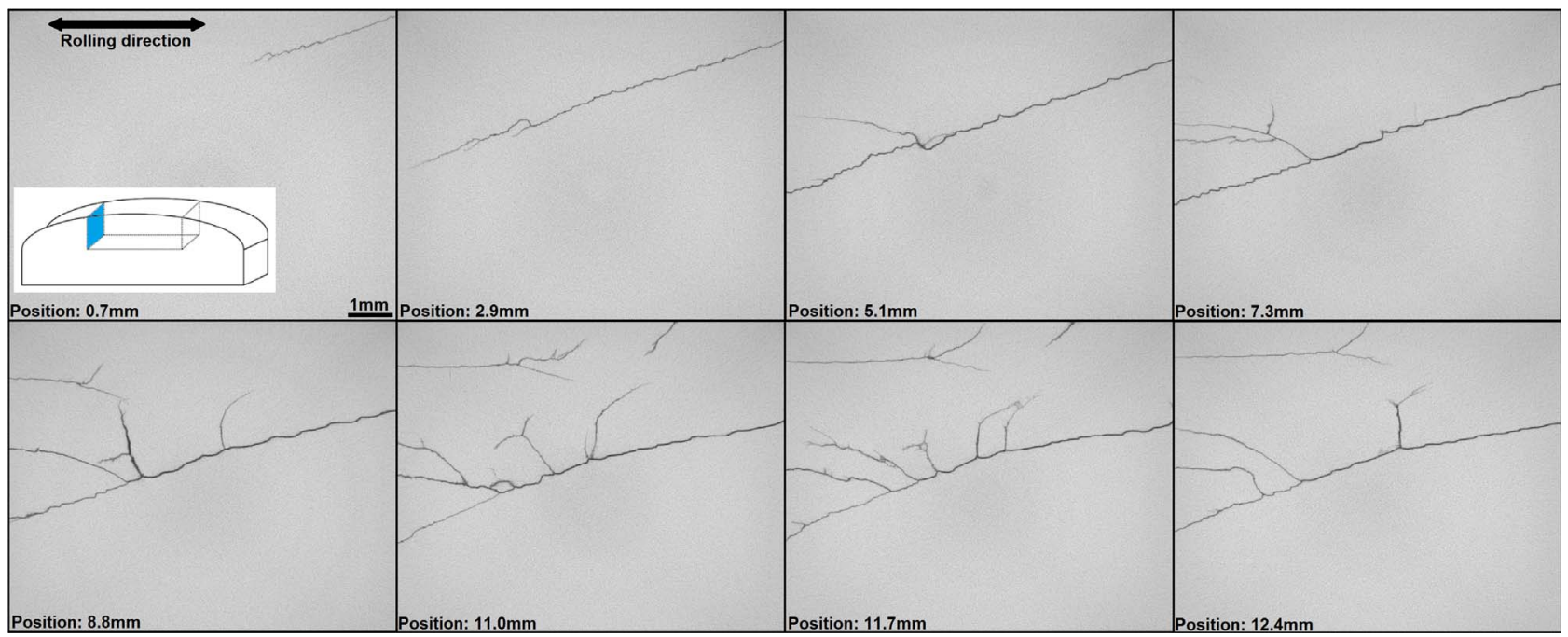

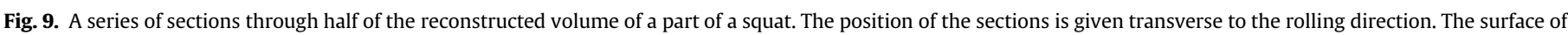

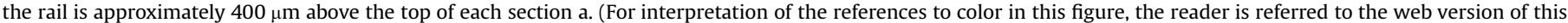
article.)
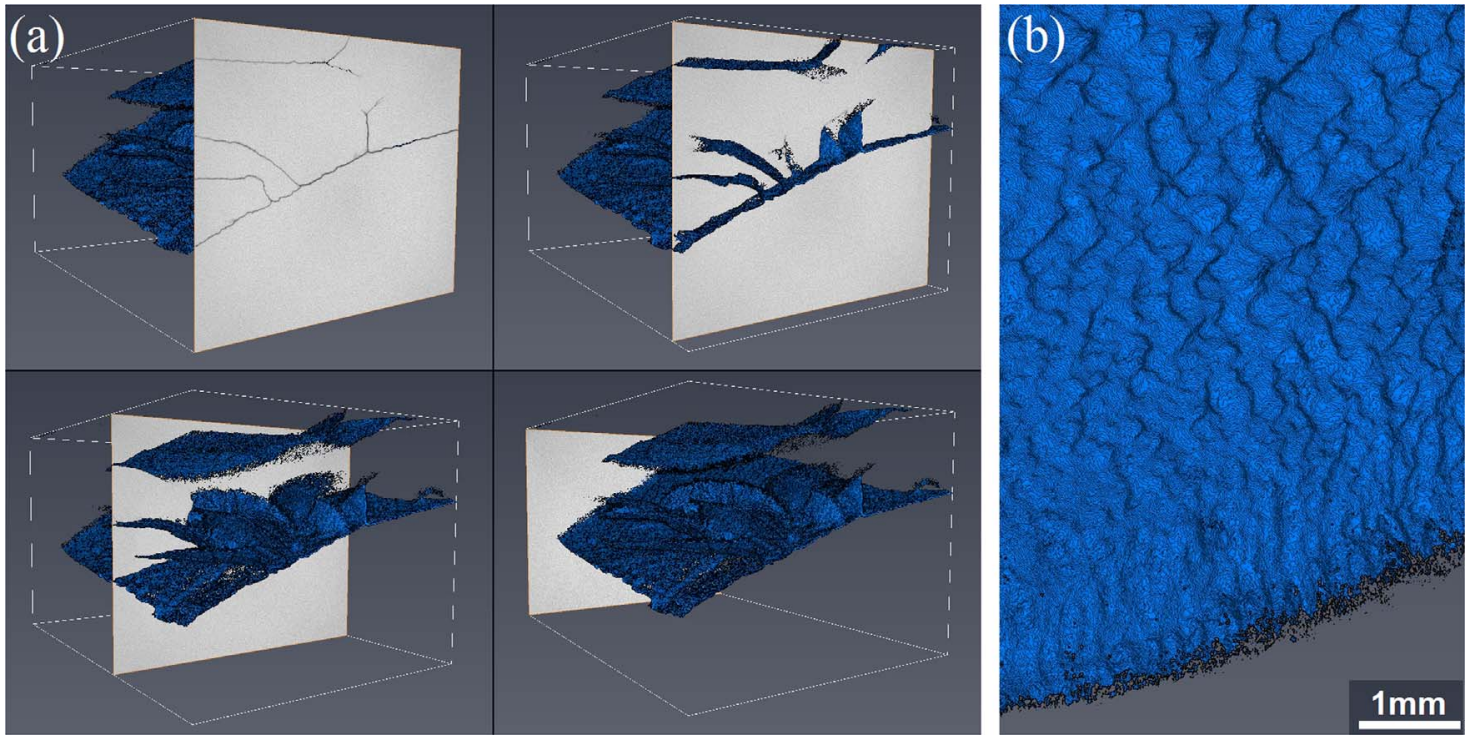

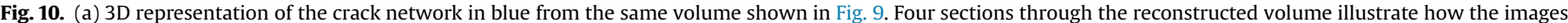

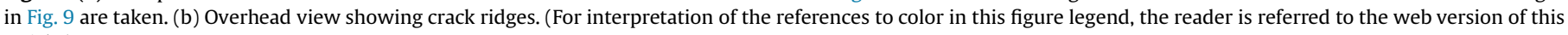
article.)

in Fig. 7c. Closer to the tip, the opening of the crack may be too narrow for detection with the given resolution. Side branches can be hidden due to limited angles; they never become parallel to the radiation. The surface obtained through the reconstruction is compared to that from metallography in Fig. 6. Although the depth can be estimated, the direction of the crack close to the crack tip provides important information on the severity of the squat. This means the detection in-field is always one step behind, which is a severe drawback when predicting whether the squat will cause further damage, such as transverse fracture or surface shelling. Perhaps this can be solved by combining it with complementary NDT techniques.

The surface-breaking crack is sometimes missing from the 3D reconstruction, which may be due to the difficulty of capturing curved surfaces, or discerning it from the surface topography removed in the image treatment. And the larger the amplitude of the ridges, the less distinct the contrast in the X-ray images. It can also be observed that the reconstructed crack geometry does not extend to y-positions beyond the surface-breaking part of the crack, represented in Fig. 6. That is, the peripheral parts of the squat which lie entirely below the surface cannot be detected unless it extends to the surface, due to the tight closure which exists.

\subsection{Metallographic investigation}

The metallographic investigation was complemented with light optical microscopy (LOM), stereomicroscopy and scanning electron microscopy (SEM). This deeper look at the area within and around the crack gave better insight into the mechanisms involved in crack propagation, such as plastic deformation and thermal damage. It was found that plastic deformation has occurred, mostly around the surfaces of the crack, however it was not present along the entire length. However, deformation may exist in a 

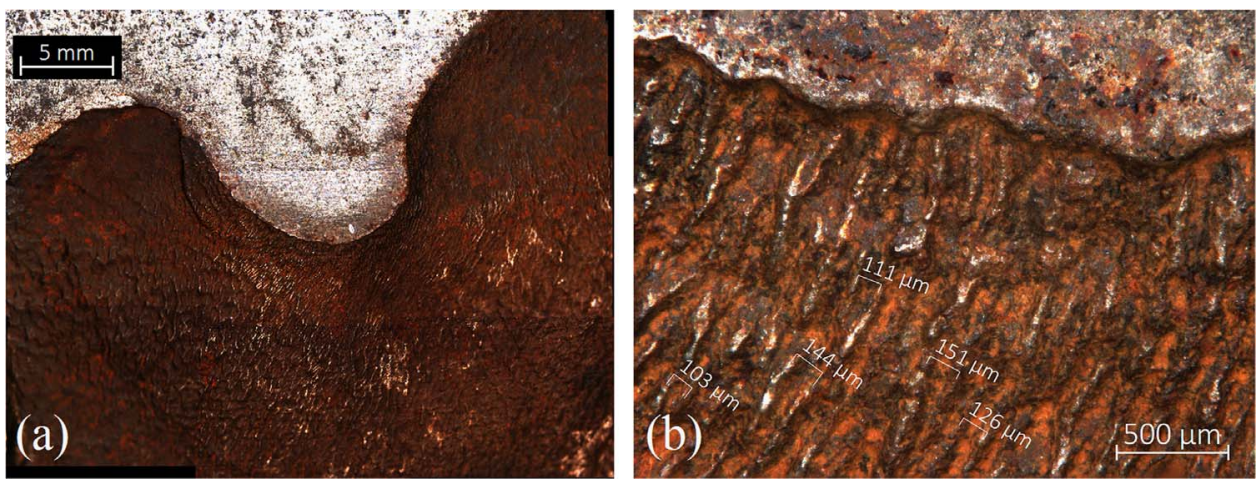

Fig. 11. Images from stereomicroscopy of (a) squat surface, and (b) detail of squat surface close to the tip of the v.
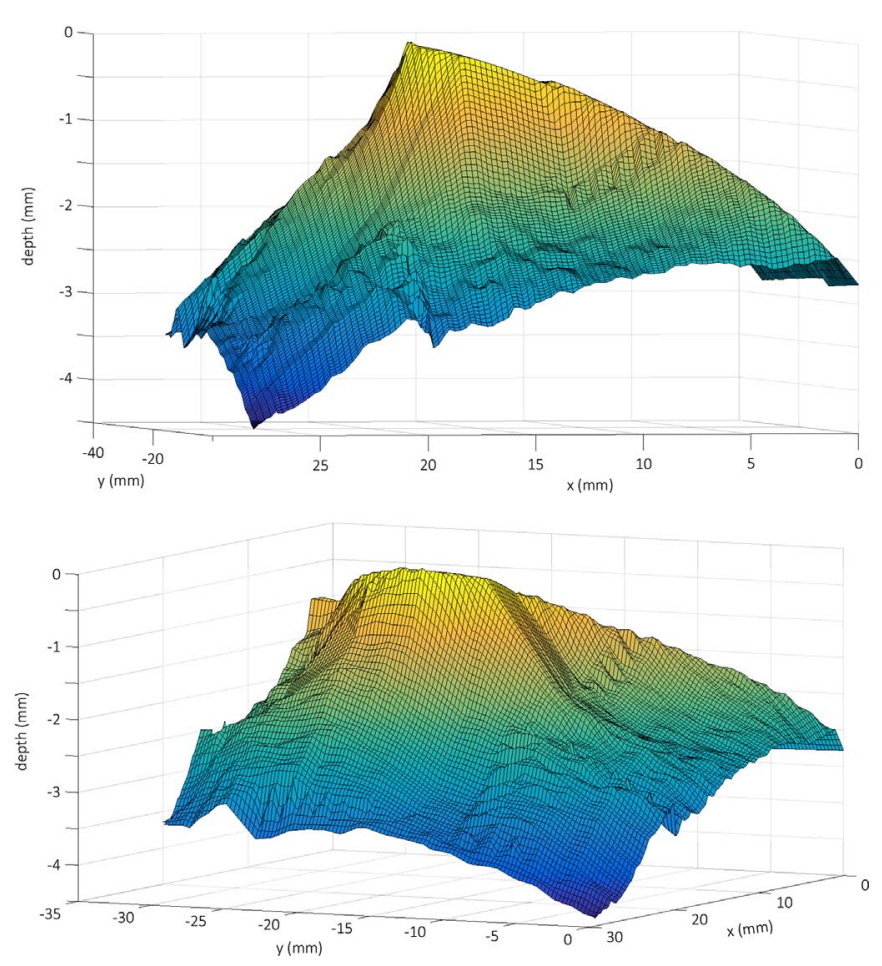

Fig. 12. Mapping of overall squat geometry, $1 \mathrm{~mm}$ step size.

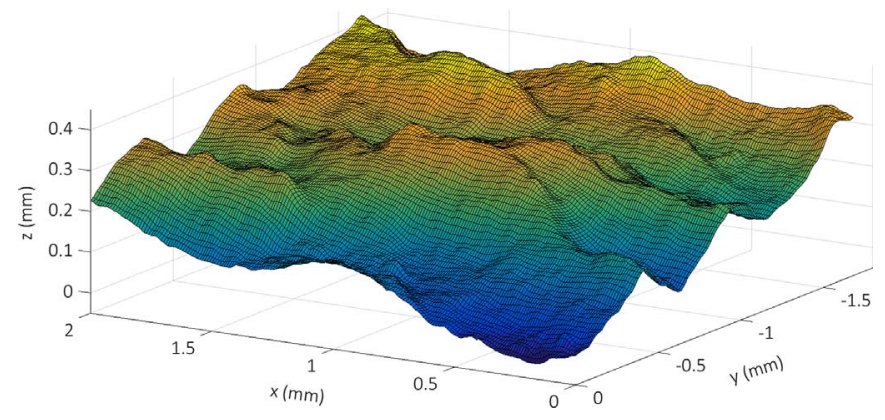

Fig. 13. Mapping from topography showing ridges, 50 um step size.

perpendicular direction. No signs of thermal damage were seen in this case.

LOM revealed the presence of branching along the main crack, which is not captured in the geometric reconstruction. These branches are filled with a residue, which was investigated using SEM. These residues within the cracks are severely deformed bulk material which has been repeatedly sheared and compacted due to the friction between crack faces [23]. The main crack tip is also often filled with these residues, which adds to the difficulty of detecting it in the radiography and reconstruction stages, since the residues have approximately the same density as the bulk material.

\subsection{X-ray tomography}

X-ray tomography requires acquisition of images from a large angle interval. Typically, images are acquired while rotating the sample $360^{\circ}$. Thus standard tomography is not a suitable technique for studying rails in-field. Another limitation is that highenergy X-rays are needed to penetrate a whole rail head. However when mm-sized samples have been cut from a rail, they can be investigated with high-resolution tomographic reconstructions and the whole crack network can be investigated in 3D. The fracture surface can be segmented and studied without opening the cracks. It is possible though that residual stresses are relieved when extracting the sample, so that crack widths change.

\subsection{Topography measurements}

A squat was broken open, and the bulk-side face of the crack network was investigated for topography. The 3D mapping showed a highly textured surface on different scales: pronounced ridges, some beach marks, and variations in surface roughness. These features are not apparent in the reconstruction nor the metallographic sectioning, adding an extra dimension to the complex squat crack network geometry. A careful investigation of the fatigue surface was not done in this study, but it should be noted that the presence of ridges signifies a relative movement between the crack faces only in the direction of the ridges. In other areas, varying sliding direction between the crack faces generates a smooth surface due to plastic deformation and wear.

\section{Conclusions}

The aim of the study was to investigate the potential of different methods to characterize the geometry of squats. The general conclusion is that the different methods are complementary, and observations made using one method can sometimes explain deficiencies of other methods.

The X-ray radiography method previously developed $[17,19]$ is beneficial in the case of truly non-destructive squat investigation, since it provides a clear visualization of the crack network below the rail surface, in a compiled series of exposures taken without any damage to the rail. A semi-automated image analysis for 3D reconstruction was developed in this study, and the results showed a squat network geometry similar to that described in 
literature. Some aspects remain to be improved in order to more accurately capture the curved surfaces of the crack planes, as well as to extrapolate the surface to deeper depths, i.e. to the crack tip. The use of radiography and image analysis proved to be sufficiently accurate for the description of the squat crack network at medium depths, however this technique could not locate the crack tip or discern branching. Although an estimation of the crack depth can made, it is the direction of the crack close to the crack tip which provides information on the severity of the squat when predicting whether the squat will cause further damage, such as transverse fracture or surface shelling. Limitations in this method exist due to the characteristics of radiography, and time-efficiency remains to be improved in all steps of the procedure in order for this method to be efficiently applied in-situ.

Metallographic investigation was done in order to compare the results from the X-ray 3D reconstruction method to the physical reality of the network, as well as take a closer look at the microstructure. Sectioning is time-consuming and destructive, but the resulting images clearly describe the characteristics of the crack at given coordinates. Microscopic investigation of the material close to and within the crack showed branching occuring along the main crack, and those branches and crack tips are filled with residues coming from deformed bulk material. Plastic deformation had occurred on the surface of the rail as well as along the crack, however evidence is not present everywhere, indicating other mechanisms drive the crack growth in this case. No white etching layer (WEL) or thermal damage were apparent.

X-ray tomography allows visualization of the crack network with high-resolution in mm-sized samples. This is a superior characterization method compared to radiography in terms of sensitivity to network geometry. That is, both the main crack and all branches can be studied in high detail in the tomographic reconstructions of the sample and the technique gives access to the fracture surface without physically opening the cracks.

Topography measurements provide a 3D mapping of the surface of a similar squat network. In terms of characterizing the main crack topography, this method was able to differentiate many different magnitudes of surface texture which exist. Though this method required partial destruction of the specimen, a good visualization of the main crack was easily achieved, and there was possibility of gathering additional information about the surface roughness, which could not be measured using the other methods.

\section{Acknowledgment}

The current work is part of activities within the Swedish National Centre of Excellence CHARMEC (Chalmers Railway Mechanics) with additional support from the Vinnova verification for growth programme. Banedanmark is acknowledged for providing the rail section for X-ray tomography.

\section{References}

[1] S.L. Grassie, Squats and squat-type defects in rails: the understanding to date, J. Rail Rapid Transit 226 (2011) 235-242.

[2] A. Ekberg, B. Åkesson, E. Kabo, Wheel/rail rolling contact fatigue - probe, predict, prevent, Wear 314 (1-2) (2014) 2-12.

[3] H.M. Tournay, J.M. Mulder, The transition from the wear to the stress regime, Wear 191 (1-2) (1996) 107-112.

[4] W. Daniel, S. Pal, M. Farjoo, Rail squats: progress in understanding the Australian experience, J. Rail Rapid Transit 227 (5) (2013) 481-492.

[5] M. Steenbergen, R. Dollevoet, On the mechanism of squat formation on train rails- part I: origination, Int. J. Fatigue 47 (2013) 361-372.

[6] Z. Li, Squats on railway rails: Wheel-rail Interface Handbook, no. 13, 2009, pp. 409-436.

[7] S.L. Grassie, D.I. Fletcher, E.A. Gallardo-Hernandez, P. Summers, Studs: a squattype defect in rails, J. Rail Rapid Transit 226 (2011) 243-256.

[8] J. Smulder, Management and research tackle rolling contact fatigue, Railw. Gaz. Int. (2003) 439-442.

[9] Z. Li, X. Zhao, C. Esveld, R. Dollevoet, M. Molodova, An investigation into the causes of squats- Correlation analysis and numerical modeling, Wear 265 (2008) 1349-1355.

[10] H.C. Eden, J.E. Garnham, C.L. Davis, Influential microstructural changes on rolling contact fatigue crack initiation in pearlitic rail steels, Mater. Sci. Technol. 21 (6) (2005) 623-629.

[11] S.L. Wong, P.E. Bold, M.W. Brown, R.J. Allen, A branch criterion for shallow angled rolling contact fatigue cracks in rails, Wear 191 (1996) 45-53.

[12] R. Pohl, A. Erhard, H.-J. Montag, H.-M. Thomas, H. Wüstenberg, NDT techniques for railroad wheel and gauge corner inspection, NDT E Int. 37 (2004) 89-94.

[13] M.P. Papaelias, C. Roberts, C.L. Lewis, A review on non-destructive evaluation of rails: state-of-the-art and future developments, J. Rail Rapid Transit (2008) 367-384.

[14] H. Jagannathan, N. Bhaskar, P.C.N. Sriraman, N.A. Vijay, A step towards automatic defect pattern analysis and evaluation in industrial radiography using digital image processing, in: Proceedings of the 15th World Conference on Nondestructive Testing, 2000.

[15] R. Patel, Digital applications of radiography, in: 3rd Middle East Nondestructive Testing Conference \& Exhibition, Bahrain, Manama, 2005.

[16] L. Hammar, H. Wirdelius, Radiographic sensitivity improved by optimized high resolution X-ray detector design, in: Proceedings of the International Symposium on Digital Industrial Radiology and Computed Tomography, Lyon, France, 2007.

[17] L. Hammar,Novel design of high resolution imaging X-ray detectors, in: Proceedings of the 18th World Conference on Nondestructive Testing, Durban, South Africa, 2012.

[18] U. Ewert, U. Zsherpel, M. Jechow, Essential parameters and conditions for optimum image quality in digital radiology, in: Proceedings of the 18th World Conference on Nondestructive Testing, Durban, South Africa, 2012.

[19] C. Jessop, J. Ahlström, L. Hammar, 3D Characterization of squat crack network using high-resolution X-ray radiography, in: Proceedings of the 35th Riso International Symposium on Materials Science: New Frontiers of Nanomaterials, 2014, pp. 339-348.

[20] I. The MathWorks, MATLAB and Statistics Toolbox Release 2014b, Natick, Massachusetts, United States, 2014.

[21] S. Simon, A. Saulot, C. Dayot, X. Quost, Y. Berthier, Tribological characterization of rail squat defects, Wear 297 (2013) 926-942.

[22] A. Hohenwarter, R. Pippan, Fracture and fracture toughness of nanopolycrystalline metals produced by severe plastic deformation Subject Areas Article number 20140366, Philos. Trans. R. Soc. A Math. Phys. Eng. Sci. 373 (2038) (2015).

[23] K. Cvetkovski, J. Ahlström, M. Norell, C. Persson, Analysis of wear debris in rolling contact fatigue cracks of pearlitic railway wheels, Wear 51-56 (2014). 